\title{
Actitudes lingüísticas de estudiantes universitarios hacia el quechua en Cusco
}

\author{
Yuliana Kenfield $^{1 \mathrm{a}}$, Wenceslao Huayllani Mercado² y Emely Yexy Huillca Quishua ${ }^{3}$ \\ Universidad San Antonio Abad del Cusco, Perú123 \\ Orcid ID: https://orcid.org/0000-0001-8373-55281 \\ Orcid ID: https://orcid.org/0000-0002-2277-35872 \\ Orcid ID: https://orcid.org/0000-0001-8373-55283
}

\section{Recibido: 21 de junio de 2017}

Aceptado: 01 de diciembre de 2017

\begin{abstract}
Resumen
La ciudad del Cusco, antigua capital de la civilización Inca, alberga por más de tres siglos a la Universidad Nacional San Antonio Abad del Cusco (UNSAAC). Estudiar las actitudes lingüísticas de los estudiantes universitarios hacia el quechua se hace necesario para entender el posible escenario para la mantención y recuperación del idioma quechua en el Cusco, en particular en la UNSAAC la cual tiene una creciente población estudiantes quechuahablante. En este artículo nos interesa explorar las actitudes lingüísticas de los estudiantes universitarios hacia el quechua por medio de una encuesta anónima no probabilística. Para el propósito de este articulo entendemos la actitud lingüística como la respuesta afectiva de los estudiantes de la UNSAAC hacia el idioma quechua. En el 2016 más de 300 estudiantes de la UNSAAC fueron encuestados para observar las actitudes lingüísticas hacia el quechua. La hipótesis que se logró comprobar fue la siguiente: Los estudiantes universitarios de la UNSAAC demuestran actitudes linguiísticas positivas hacia el quechua. A diferencia de la conclusión de Wolck sobre que la lengua quechua tiende a tener un alto valor positivo a nivel personal y no así un alto valor institucional instrumental (Wolck, 2003); los resultados de este estudio nos revelan la existencia del valor tanto personal e institucional del quechua adscrito por los estudiantes ya que sus actitudes lingüísticas son positivas hacia el quechua fuera y dentro de la universidad.
\end{abstract}

Palabras clave: Actitudes Lingüísticas, el Idioma quechua, sociolingüística Andina, el quechua en la universidad

\section{Language Attitudes of College Students towards Quechua in Cusco}

\author{
Abstract \\ ${ }^{\text {a }}$ Correspondencia al autor \\ E-mail: yulianasi@hotmail.com
}


The city of Cusco, former capital city of the Inca Civilization, has housed for over three centuries the Universidad National San Antonio Abad of Cusco (UNSAAC). Study of the linguistic attitudes of college students towards Quechua becomes necessary to understand the potential landscape for the maintenance and recovery of the Quechua language in Cusco, particularly at the UNSAAC which has a growing Quechua-speaking population. This article explores the linguistic attitudes of university students towards Quechua through an anonymous, non-probabilistic survey study. Herein, languaje attitude is understood as the affective response of the students of the UNSAAC towards the Quechua language. In 2016, more than 300 UNSAAC students were surveyed to observe their languaje attitudes towards Quechua. The hypothesis tested was the following: University students of the UNSAAC demonstrate positive languaje attitudes towards Quechua. Unlike Wolck's conclusion that the Quechua language tends to have a high positive value on a personal level butnot a high instrumental institutional value (Wolck, 2003); the results of this study reveal the existence of the personal and institutional value of Quechua held by students as their languaje attitudes are positive towards Quechua both outside and within the university.

Key-words: Language Attitudes, the Quechua language, Andean sociolinguistics, Quechua at the university

\section{Introducción}

La diversidad lingüística y cultural peruana ha sido y sigue siendo un gran reto para el estado peruano, ya que uno de sus anhelos es fortalecer el uso del quechua en el país y practicar diálogos reales con los pueblos quechuas (Hornberger, 1998).

La región del Cusco tiene un interés particular por la preservación y difusión del idioma quechua debido a su legado histórico, no solo Inka, sino también pre-inca el cual aún es muy latente en la región tanto en espacios urbanos como rurales.

Pensamos que un primer paso para entender la mantención de la presencia lingüística quechua son las actitudes lingüísticas entendidas como respuestas afectivas (Crystal, 1997) al estatus social del idioma quechua en la universidad y sociedad. Entender el status que una persona o sociedad le entrega al quechua puede ser un paso importante para comprender la disposición de la población universitaria hacia la continuidad linguiística quechua.

En el campo específico de las actitudes lingüísticas hacia el quechua en comparación con el castellano, el trabajo de Wolck (1973) y Van Gleish \& Wolck (1994) surgió en el contexto peruano que llevaba en su agenda educacional el bilingüismo quechua-castellano para la nación. La investigación longitudinal de Wolck en actitudes linguiísticas representa el trabajo más ambicioso en Perú. En 1973, durante el surgimiento de políticas de educación bilingüe en el Perú, se utilizó la investigación sobre la actitud hacia el lenguaje para evaluar el estatus social de un idioma sobre el otro. 
El análisis dicotómico inherente llevó a Wolck a evaluar las actitudes hacia el quechua y el castellano en un marco conceptual binario. Wolck separó las actitudes lingüísticas como hispanista - promoviendo el proyecto de hispanización colonial - e indigenista - promoviendo la retención de la lengua quechua (Ryan y Giles, 1982). La investigación de Wölck reveló que, a medida que aumentaba el bilingüismo, las diferencias de estatus entre el castellano y el quechua disminuían. Sin embargo, Wolck también sostuvo que "mientras las lenguas minoritarias evocan reacciones afectivas personales más positivas, las lenguas mayoritarias llevan más valor institucional instrumental" (Wölck 2003: 31 y 36).

Esta última conclusión se vuelve problemática ya que parece limitar el destino de lenguas como el quechua en la educación superior. Para cuestionar esta limitación potencial, la investigación de encuesta exploró las actitudes de estudiantes universitarios de la UNSAAC hacia la presencia del quechua en su ambiente universitario. El presente estudio nos acercará hacia las actitudes lingüísticas de los estudiantes universitarios para poder explorar la valoración que ellos le dan al quechua en la universidad en Cusco.

\section{Metodología}

En el diseño de este estudio se empleó una muestra representativa no probabilística debido a que no todos los estudiantes universitarios de la Universidad Nacional San Antonio Abad del Cusco [UNSAAC] fueron invitados a tomar la encuesta. Se invitaron a tomar la encuesta a más de mil estudiantes (de los 15,000) de manera personal y electrónica. Lamentablemente no pudimos acceder a mas aulas universitarias debido a que la UNSAAC en el semestre 2016-I fue tomada por los estudiantes por más de tres semanas. El cuestionario fue accesible de dos maneras: en línea por medio de un enlace del servidor SurveyMonkey compartido vía correo electrónico (por preferencia de los estudiantes), y en papel compartido por medio de sus docentes universitarios in situ. La participación de los estudiantes en esta encuesta fue voluntaria, anónima y confidencial. Para ser elegibles para su inclusión en el estudio, los estudiantes universitarios tenían que estar inscritos en el semestre 2016-I y ser mayores de edad. En la encuesta participaron estudiantes de 21 diferentes carreras universitarias del total de las 32 carreras que ofrece la UNSAAC (Anuario N 29, 2015). Entre las 21 carreras se hallaban carreras de humanidades como historia, de ingenierías como ingeniería metalúrgica, y carreras de ciencias de la salud como medicina humana. 


\section{El instrumento}

La encuesta que se usó en este estudio es una adaptación de los cuestionarios sociolingüísticos de Manley (2008) y Bekker (2004). En general el cuestionario tiene tres secciones: a) datos demográficos, b) uso del lenguaje, c) actitudes lingüísticas. Para el presente artículo nos centraremos en la sección c: actitudes lingüísticas, y tomaremos el dato demográfico sobre la lengua materna. El cuestionario contiene una escala Likert para examinar las actitudes lingüísticas. En esta escala se presentó cuatro opciones: a) Estoy completamente de acuerdo, b) Estoy de acuerdo, c) Estoy en desacuerdo, y d) Estoy totalmente en desacuerdo. No usamos una escala binaria ya que deseábamos apreciar mejor el tipo de respuesta afectiva de los estudiantes universitarios hacia el quechua, por lo cual teniendo cuatro opciones el estudiante podía encasillar su valoración de manera menos restringida. La encuesta sociolingüística fue completada por 303 estudiantes, resultando en un margen de error del 5\% basado en una población estudiantil universitaria de 15,000 en UNSAAC (Anuario N29, 2014).

En particular, la utilización de una escala tipo Likert fue usada para explorar las actitudes lingüísticas. En esta escala los estudiantes leyeron declaraciones acerca del estatus social del quechua en la universidad. Las cuatro declaraciones que hemos usado para el análisis estadístico para este articulo son las siguientes: a) El idioma quechua está a la misma altura del castellano, b) En general los jóvenes que saben idiomas originarios Andinos quechuas deberían usar sus idiomas entre ellos nomás y fuera de la Universidad, c) En general los jóvenes que saben el idioma quechua deberían usarlo dentro de la Universidad para que los demás aprendan (si no lo conocen) o practiquen (si en caso lo conocen) el quechua. d) La universidad no debería fomentar el uso del quechua por ser un retraso.

Las declaraciones incluidas en la escala se enfocaron principalmente en observar las actitudes linguiísticas de los estudiantes hacia el quechua académico, el quechua como idioma indígena, el quechua en la universidad.

En este artículo solamente se discutirán las creencias sobre el valor del idioma quechua en la universidad, y en la sociedad. Deseamos acercarnos no solo a las actitudes lingüísticas hacia el quechua en general, sino en específico a la mirada que le dan los estudiantes universitarios al quechua dentro de la universidad.

Tamaño de muestra 
El tamaño de muestra fue determinado por la formula descrita a continuación:

Margen: $5 \%$

Nivel de confianza: $95 \%$

Población: 15000

$$
\begin{aligned}
& \text { Ecuacion Estadistica para Proporciones } \\
& \text { poblacionales } \\
& \mathrm{n}=\text { Tamaño de la muestra } \\
& \mathbf{z}=\text { Nivel de confianza deseado } \\
& n=\frac{z^{\wedge} 2\left(p^{\star} q\right)}{e^{\wedge} 2+\frac{\left(z^{\wedge} 2\left(p^{\star} q\right)\right.}{N}} \\
& p=\text { Proporcion de la poblacion con la caracteristica } \\
& \text { deseada (exito) } \\
& q=\text { Proporcion de la poblacion sin la caracteristica } \\
& \text { deseada (fracaso) } \\
& \mathrm{e}=\text { Nivel de error dispuesto a cometer } \\
& \mathrm{N}=\text { Tamaño de la poblacion }
\end{aligned}
$$

dando como resultado el tamaño de muestra de 350 estudiantes a ser encuestados.

\section{Aplicación del instrumento}

La encuesta fue completada por los estudiantes universitarios durante los meses de junio a setiembre del año 2016. Esta encuesta fue completada por los estudiantes universitarios tanto en línea, por medio de una invitación a través de un enlace en línea distribuida a la lista de correo electrónico de los estudiantes universitarios, al igual que por el medio tradicional de entregar las encuestas en papel. Los estudiantes que participaron en la encuesta lo hicieron en su tiempo disponible. Quienes llenaron el cuestionario de papel lo colocaban en un sobre (no necesariamente el mismo día en el que conocieron sobre la encuesta) para que el equipo de investigadores lo recogiera en días posteriores. Debido a la falta de acceso fiable del internet en la ciudad del Cusco, los estudiantes investigadores fueron responsables de la administración de la encuesta en forma tradicional en algunas de las aulas universitarias.

\section{Análisis}

Los resultados del estudio fueron analizados a través de métodos de estadística descriptiva por conteo. Por lo tanto, el enfoque del análisis es descriptivo, pero no predictivo. La utilización del programa Excel 2016 ayudó en la etapa de análisis de datos. 
En general, los análisis estadísticos corroboraron la hipótesis planteada: Los estudiantes universitarios de Cusco demuestran actitudes lingüísticas positivas hacia el quechua. Los indicadores utilizados para observar las actitudes lingüísticas fueron las valoraciones de la escala Likert. Las valoraciones dadas a las declaraciones analizadas para este artículo estarán solamente enfocadas a las actitudes hacia el quechua dentro de la universidad sino al quechua en general.

\section{Resultados}

\section{Lengua materna de los estudiantes}

Primeramente, el resultado sobre la primera lengua de los estudiantes universitarios puede ser visualizado en el grafico uno. Esta obtención de datos sobre la primera lengua son datos que no fueron antes documentados, al menos esos datos específicos no son hallados en los datos estadísticos de los anuarios publicados por la Universidad San Antonio Abad sobre la demografía de los estudiantes.

Al observar los porcentajes de estudiantes con la lengua materna quechua hallamos que más del 32.29\% la tienen como lengua materna. Interesantemente también hallamos un 3.14\% de estudiantes que declaran tener el bilingüismo quechua-castellano como primera lengua, y un $64.57 \%$ tienen como lengua materna el castellano. En particular, hallamos interesante el hecho que los encuestados eligieran dos lenguas como su lengua materna. El formato de la encuesta dio libertad al encuestado a elegir más de una lengua como materna, este formato fue deliberado de esta manera ya que aceptamos el hecho de que las personas pueden crecer con el bilingüismo desde la infancia. El hecho de conocer el porcentaje de bilingües quechua-castellano de nacimiento refleja la contraposición del estudio realizado por Wolck $(1973,1994)$ que invisibilizaba la cantidad de bilingües de nacimiento. Estos resultados están relacionados con la realidad lingüística de la región del Cusco que es conocida por su alto número de quechuahablantes, en particular en provincias del Cusco.

\section{Actitudes lingüísticas hacia el quechua}

Los siguientes resultados en este artículo se centran en cuatro de las diecisiete declaraciones que conformaron la escala Likert incluida en la encuesta del 2016-I. De manera general por conteo, como lo ilustra la tabla $1 \mathrm{y}$, y por porcentajes como lo muestran los gráficos 2-5, se puede observar que las actitudes y las opiniones son positivas de los estudiantes tanto al 
quechua en general, y al quechua dentro de la Universidad. Estos resultados corroboran la hipótesis planteada de este estudio, la cual reza: Los estudiantes universitarios de la UNSAAC demuestran actitudes lingüísticas positivas hacia el quechua

Tabla 1

Observación general de las respuestas

\begin{tabular}{|c|c|c|c|c|}
\hline Declaraciones Relacionadas al Quechua & $\begin{array}{l}\% \text { Completa- } \\
\text { mente de } \\
\text { acuerdo }\end{array}$ & $\%$ De acuerdo & $\begin{array}{l}\% \text { En } \\
\text { desacuerdo }\end{array}$ & $\begin{array}{l}\% \text { En total } \\
\text { desacuerdo }\end{array}$ \\
\hline $\begin{array}{l}\text { a. El idioma quechua está a la mis ma altura del } \\
\text { castellano }\end{array}$ & $49.00 \%$ & $37.00 \%$ & $11.00 \%$ & $3.00 \%$ \\
\hline $\begin{array}{l}\text { b. En general los jóvenes que saben quechuas } \\
\text { deberían usar su idioma entre ellos nomás y fuera de la } \\
\text { Universidad. }\end{array}$ & $4.60 \%$ & $21.10 \%$ & $37.30 \%$ & $37.00 \%$ \\
\hline $\begin{array}{l}\text { c. Los jóvenes que saben quechua deberían hablarlo } \\
\text { dentro de la universidad para que los demás lo } \\
\text { aprendan o practiquen. }\end{array}$ & $42.24 \%$ & $45.54 \%$ & $10.56 \%$ & $1.65 \%$ \\
\hline $\begin{array}{l}\text { d. La universidad no debería fomentar el uso del } \\
\text { quechua por ser un retraso }\end{array}$ & $3.30 \%$ & $4.60 \%$ & $27.50 \%$ & $64.60 \%$ \\
\hline
\end{tabular}

Fuente: propia

En la tabla 1 se puede apreciar el conteo de la población general encuestada (sin especificar el idioma materno) y sus valoraciones a las cuatro declaraciones.

De acuerdo a los resultados del ítem $a$ "El idioma quechua está a la misma altura del castellano" (ver gráfico uno) podemos observar que la mayoría (86\%) de los estudiantes universitarios opinan que el quechua tiene el mismo estatus social que el castellano, solamente 14\% opinan lo contrario. En la escala Likert tenemos que 49\% de los estudiantes están extremadamente de acuerdo con darle una valoración positiva al estatus del quechua frente al castellano, en comparación solo un 3\% estuvo totalmente en desacuerdo. Además, al analizar el conteo en la tabla número 1 podemos hasta apreciar que la población estudiantil considera que el idioma quechua está la misma altura del castellano.

Gráfico 1

Primera lengua de los estudiantes universitarios 


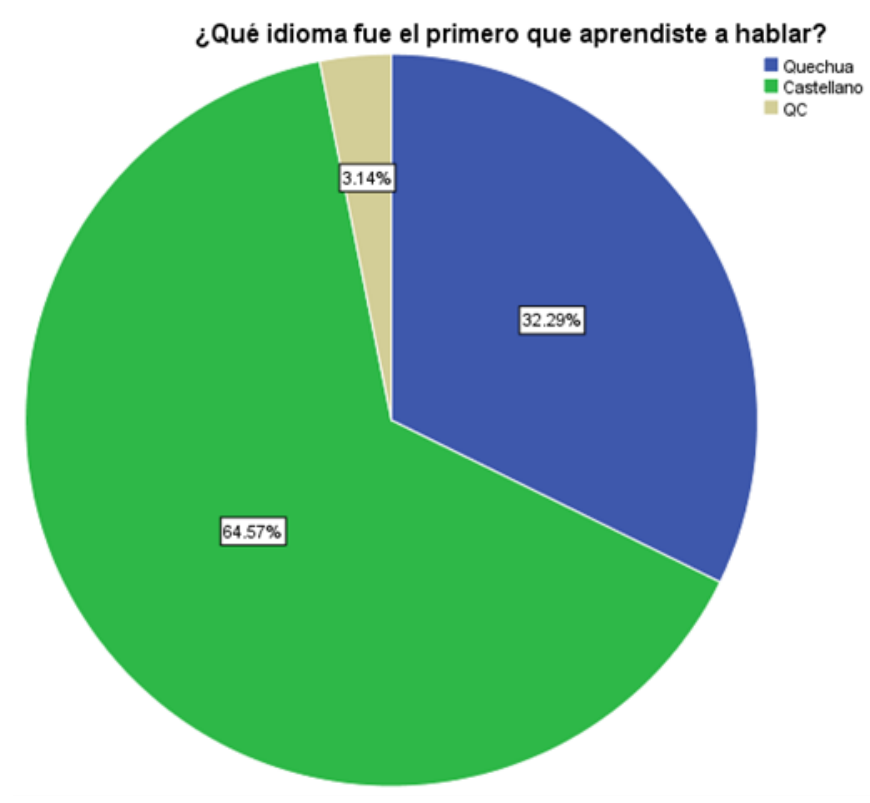

Estos resultados podrían ser explicados por medio del contexto sociocultural de la región y la universidad. En la región del Cusco se han realizado diversas medidas políticas y actividades (Yataco, 2012) enfocadas al realce del idioma e identidad cultural quechua. Algunos ejemplos de estas medidas son la Ordenanza Regional N 115-2016 sobre la inclusión obligatoria del quechua como un curso de lengua en las escuelas de la provincia del Cusco, la implementación del modelo de educación intercultural bilingüe en la región, concursos folclóricos de música en quechua organizados por la municipalidad del Cusco. Estas medidas probablemente tuvieron sus orígenes no solo en el indigenismo (Lauer, 1997), sino también en los movimientos sociales campesinos de la región Cusco pre-reformas agrarias (Pacheco, 2007; Supa Huaman, 2010; Blanco, 1974) y la industria creciente turística de la región (Perez, 2006). Otros ejemplos concretos de estas medidas se reflejan en el cambio de nombres de las calles del centro histórico del Cusco, nombres coloniales fueron reemplazados por nombres quechuas; la escenificación de diversas festividades de posible origen Inka como el Inti Raymi, Cocha Raymi (Perez, 2006), y otros que se escenifican únicamente en el idioma quechua; y la creación de la Academia Mayor de la Lengua Quechua (CoronelMolina. 2007).

Gráfico 2

El idioma quechua está a la misma altura del castellano 


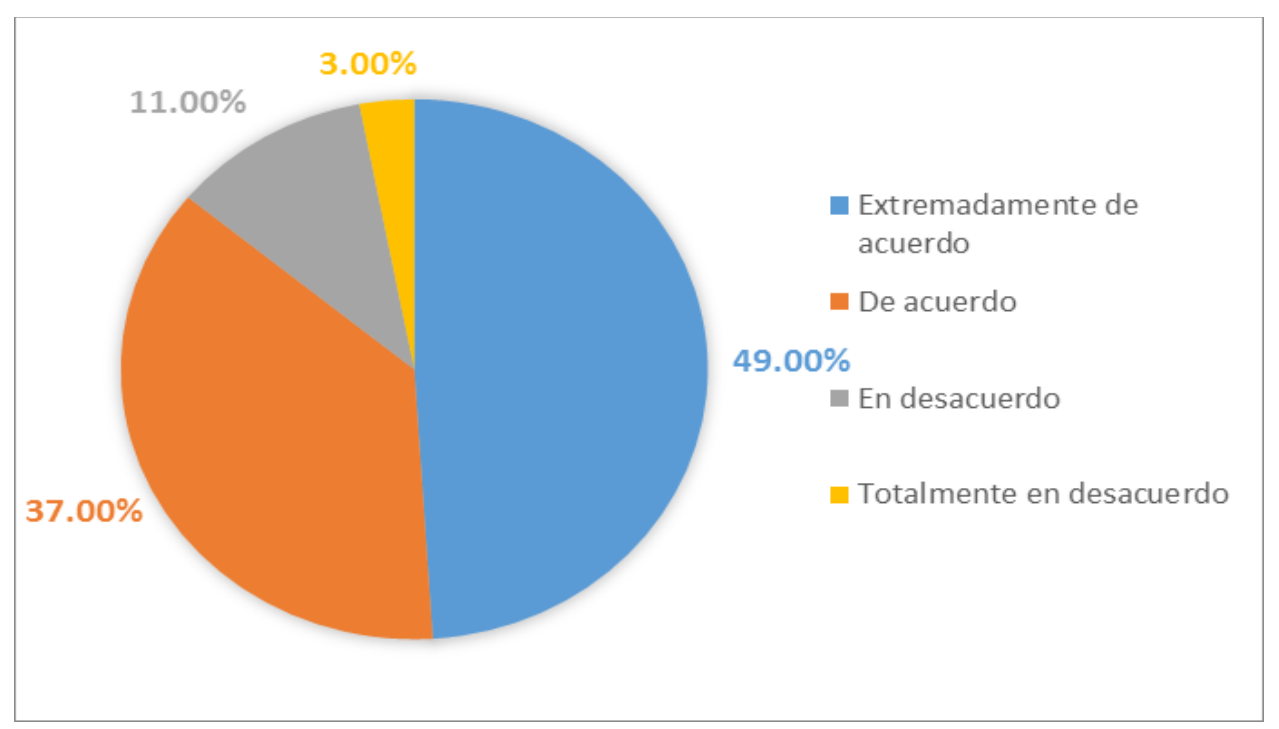

También observamos que en la declaración $a, 147$ estudiantes están completamente de acuerdo con qué el idioma quechua está a la misma altura del castellano, sólo nueve participantes están completamente en desacuerdo. Pero este conteo también cambia si es que nos ponemos a observar los resultados de la declaración $b$ "En general los jóvenes que saben quechuas deberían usar su idioma entre ellos nomás y fuera de la Universidad" el cual es más específico al quechua dentro de la Universidad. Al observar los resultados del ítem $b$ (ver gráfico 3) podemos darnos cuenta que las actitudes de estos estudiantes en la universidad cusqueña son muy positivas hacia el quechua.

A diferencia de los estudios realizados sobre actitudes hacia el quechua en el Perú mayormente han revelado el fenómeno de diglosia (Coronel-Molina, 2007, wolk, 1973). Concibiendo así al Castellano como la lengua para la movilidad social para el progreso y al quechua para la parte afectiva y no como un sinónimo de progreso sino sinónimo de un pasado el presente estudio nos ilustra un argumento diferente.

\section{Gráfico 3}

Declaración "b” Los jóvenes que saben quechua deberán hablarlo entre ellos 


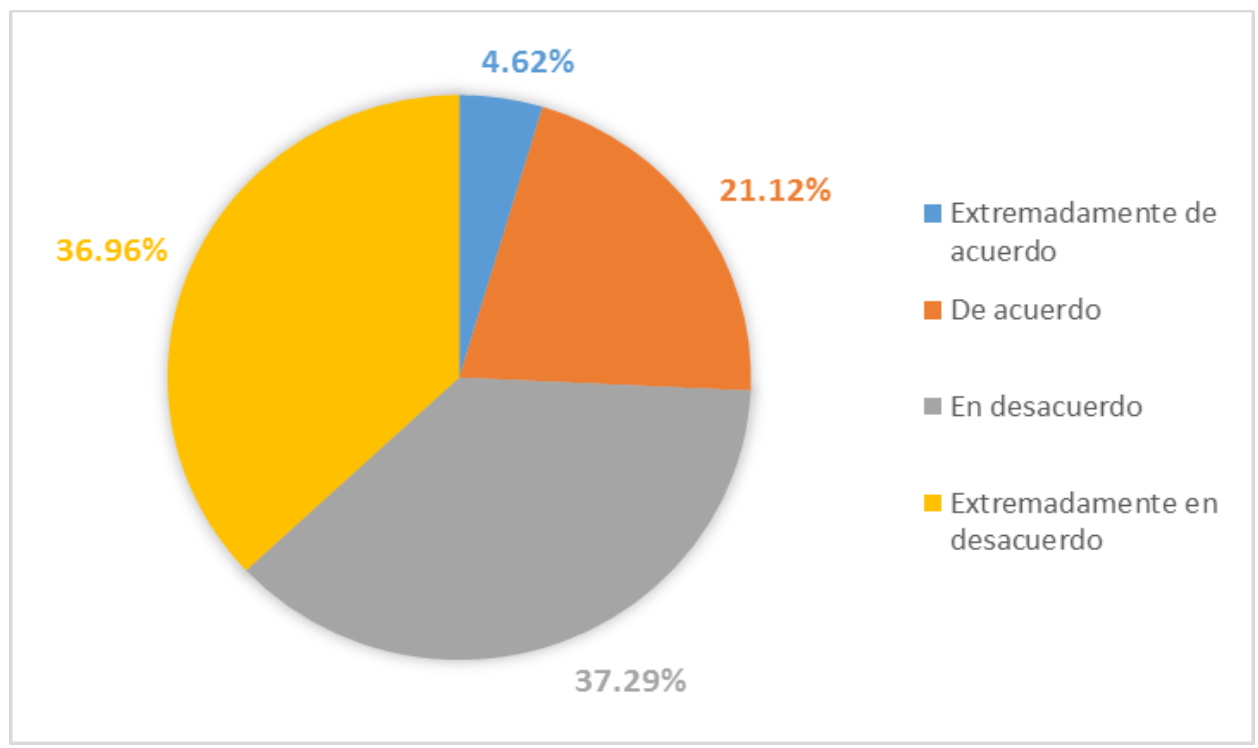

Viendo los resultados representados en el grafico tres nos damos cuenta que el $37.29 \%$ de los estudiantes universitarios está en desacuerdo que el quechua sea hablado solo en el círculo de quechua hablantes y un $36.96 \%$ está en total desacuerdo en el mismo sentido. Esto representaría la mayoría con un total de casi $80 \%$ de estudiantes quienes opinan en desacuerdo con la idea de segregar la práctica lingüística o uso del idioma quechua para espacios externos a la universidad. Igualmente, para esta mayoría el quechua no debería representar una exclusividad para los quechuahablantes. Esta actitud general positiva representa una disposición de los estudiantes a no excluir al idioma quechua ni a los estudiantes quechuahablantes de los espacios universitarios.

Esta actitud positiva mayoritaria quizá tenga relación con la identidad lingüística-cultural de los estudiantes. La identidad tiene un rol importante ya que hay una creciente población de estudiantes migrantes quienes cuando llegan a la UNSAAC traen su cultura y lengua con ellos. Esta dinámica cultural de estudiantes migrantes en la universidad abre espacios para mantener y difundir su cultura y es así que se crean espacios como el centro de Danzas Universitarias, el grupo musical Estudiantina Chumbivilcana, y el Voluntariado Intercultural Hatun Nan donde expresan prácticas culturales y lingüísticas de los estudiantes.

Sin embargo, observamos aún un porcentaje representativo de más del $25 \%$ de estudiantes quienes opinan que el quechua debe ser un idioma de uso exclusivo de los estudiantes bilingües quechua-castellano. Además de esta exclusividad, este $25 \%$ de estudiantes considera que el idioma quechua no pertenece al espacio universitario oficial y que debería mantenerse fuera del campus. 
Este tipo de percepción de no considerar al quechua como parte de una cultura universitaria puede ser un signo de prácticas de exclusión (Bourdieu, 1989). Si consideramos estos resultados además de la documentación del anuario N 29 sobre la población universitaria por grupo étnico "quechua" quienes representan solo el $18.67 \%$, podemos observar que la autoidentificacion con una cultura general quechua es baja. Surgen muchas preguntas en nosotros al reflexionar en esta observación ya que existen muchos factores que quizá promueven a un estudiante universitario a diferenciarse del "otro", en este caso del quechua y quechuahablante, o el caso de que un estudiante quechuahablante decida auto excluir su idioma quechua de un espacio oficial (Zavala, 2011). De acuerdo al estudio de Zavala (2011) con estudiantes quechuahablantes, los estudiantes percibían que su lengua materna, el quechua, se relaciona con su incapacidad para hablar correctamente el español. Esta ideología causa que los estudiantes intenten suprimir el uso de su idioma quechua al menos dentro de la universidad.

\section{Gráfico 4}

Declaración C. Lós jóvenes que saben quechua deberían hablarlo dentro de la universidad

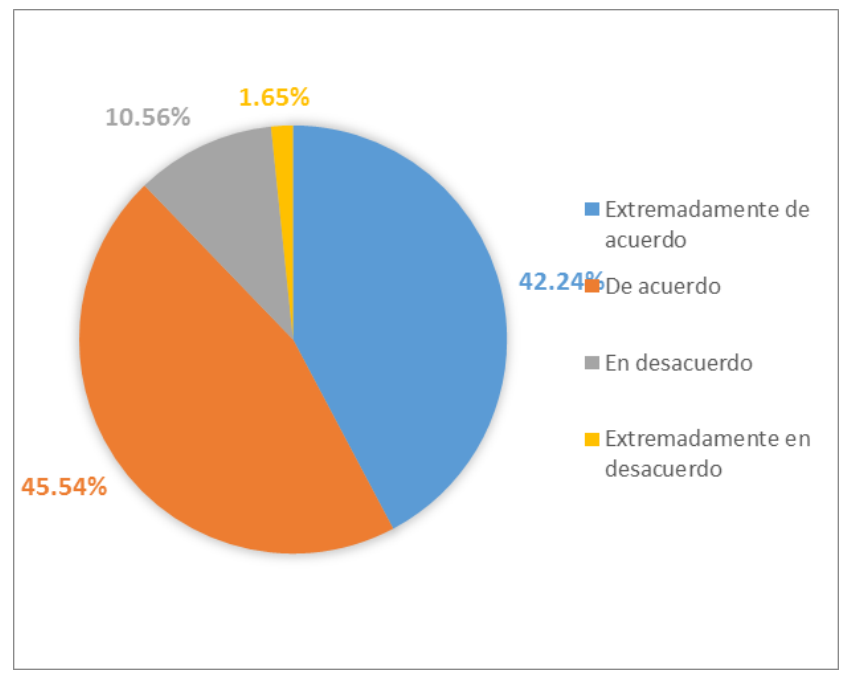

Los resultados del ítem c "En la Universidad se debe aspirar solamente a hablar un castellano correcto" (grafico cuatro) otra vez nos muestra que una mayoría, en este caso el 87.78\%, tienen una actitud positiva a que el quechua sea utilizado y difundido dentro de la universidad. En el ambiente universitario el idioma quechua es considerado una herramienta importante para muchas profesiones, por ello en diversas carreras el idioma quechua es un curso en la malla curricular (ej. comunicación, educación, historia, medicina). Por otro lado, en la Instituto de Idiomas de la UNSAAC se ofrece la certificación del idioma quechua (sitio web de Insituto de 
Idiomas CID-UNSAAC) El idioma quechua en el mencionado instituto se encuentra entre las opciones más populares hallándose en el 4to lugar después del inglés, italiano, y portugués.

Adicionalmente, el estado peruano contribuye a construir y revalorar la identidad lingüística-cultural quechua a través de los medios de comunicación, tal el caso de los canales oficiales "TV PERU y RADIO NACIONAL" lo cuales tienen noticieros en quechua creando de esa manera espacios oficiales para el quechua.

Los resultados del ítem $c$ nos hacen pensar que los estudiantes de la UNSAAC tienen esta alta valoración del idioma quechua y su valoración, podríamos decir que ahora es indispensable en el aspecto laboral, ya que muchos empleadores públicos y privadas exigen el idioma quechua como requisito indispensable para ocupar un puesto como por ej. Agronomía, enfermería, e incluso por normativa legal por parte del estado, como los que ejercen la docencia. Esto hace que los estudiantes le den valor y utilidad laboral al idioma quechua y ya no quieran mantenerlo en un círculo cerrado de solo quechuahablantes.

En general este cuadro nos muestra que hay una mirada positiva de los estudiantes a ya no mantener en un contexto cerrado el idioma quechua más bien mantenerlo y difundirlo mediante diversas formas y llevarlo a una consideración total desde el plano político dentro y fuera de la UNSAAC.

\section{Gráfico 5}

Declaración D. La universidad no debería fomentar el uso del quechua

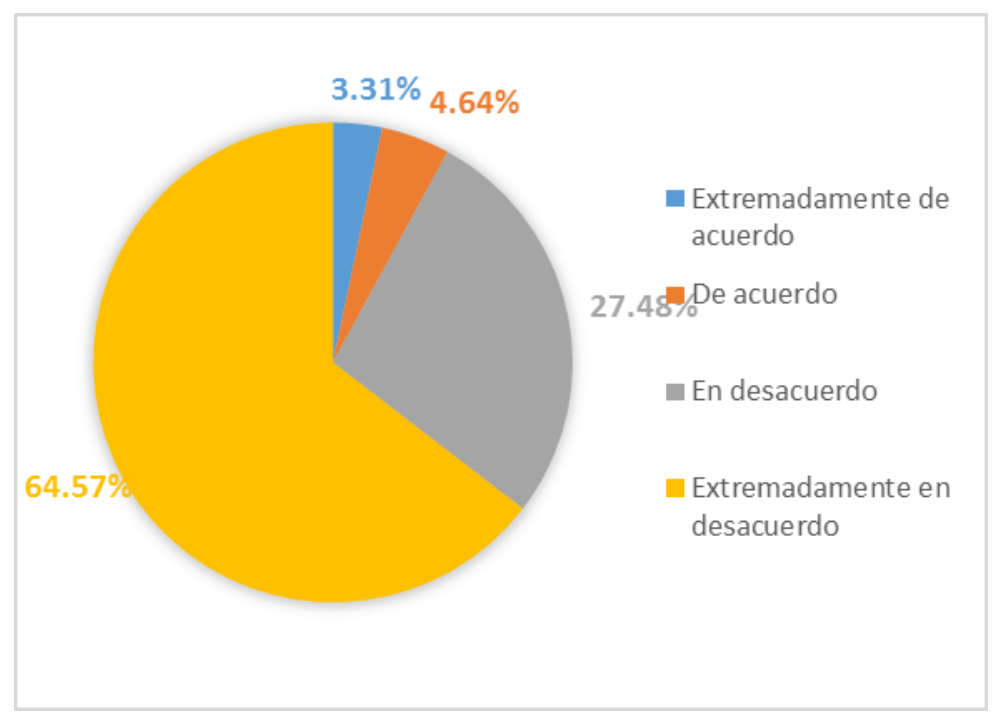


Los resultados porcentuales del ítem $d$ "La universidad no debería fomentar el uso del quechua por ser un retraso" ilustra un porcentaje, aunque bajo pero significativo, de $7.95 \%$ de la población estudiantil que rechaza fomentar el uso del quechua en la universidad por considerarlo un retraso. Una vez más, esto puede ser explicado por la ideología de mantener al castellano como lengua hegemónica y oficial en el país (Yataco, 2012). Sin embargo, aún observamos que la mayoría, 92.5\%, de la población estudiantil rechaza a afirmaciones discriminatorias como la que se presentó en la escala de Likert "La Universidad no debería fomentar el uso del quechua por ser un retraso".

Podríamos entonces inferir que estos resultados revelan una disposición de actitud hacia deconstruir el fenómeno de diglosia (Coronel-Molina, 2007) que se ha creado en un sistema que sitúa al castellano como el idioma hegemónico que cumple la mayoría de las funciones institucionales en el Perú. Esta deconstrucción se está dando por parte de los estudiantes ya que la creciente población de quechuahablantes a la par de las nuevas políticas lingüísticas regionales incentivan la práctica del uso del bilingüismo quechua-castellano en la universidad.

\section{Discusión}

Si nos ponemos a pensar en que las actitudes lingüísticas están ligadas hacia un factor que puede influenciar en la pérdida del lenguaje, entonces los datos nos están entregando buenas noticias ya que existen actitudes positivas hacia el idioma quechua por parte de los estudiantes universitarios en Cusco. Esta actitud sumada con el porcentaje de bilingües en la UNSAAC puede convertirse en un excelente escenario para el mantenimiento del quechua en el ambiente universitario. Sin embargo, se abre la necesidad de explorar aún más de manera cualitativa estas actitudes y más aún el entendimiento de cómo conciben esas actitudes los estudiantes universitarios en relación con el mantenimiento del bilingüismo y el quechua dentro de la universidad. Tal vez los esfuerzos recientes universitarios de promover la revaloración al quechua están provocando estas actitudes positivas.

La valoración positiva general de los estudiantes universitarios en el Cusco hacia el quechua demuestra que, no sólo el idioma quechua es valorizado por las afectividades personales, sino también el idioma quechua es considerado como parte de un mundo académico instrumental. 
Estas actitudes lingüísticas entonces nos estarían dando pistas quizá de un cambio, mental y social, de los quechuahablantes hacia una histórica autoexclusión lingüística.

Debido a que los resultados generales discutidos en este artículo muestran actitudes positivas de los estudiantes universitarios particularmente hacia el quechua, este equipo relaciona el resultado con políticas de revitalización del idioma, aunque aún se debe indagar más sobre las practicas reales del quechua; creemos que es necesario comparar las actitudes lingüísticas con las prácticas De igual modo sería importante hacer un seguimiento longitudinal de la compatibilidad de actitudes con los usos, especialmente en una región en la cual más del $60 \%$ de sus pobladores tienen como lengua materna el castellano (INEI, 2007).

Este estudio es parte de la primera etapa de una investigación participativa que incluye la colaboración entre estudiantes de pregrado de la Universidad Nacional San Antonio Abad del Cusco y una estudiante de posgrado de la Universidad Nuevo Mexico.

\section{Referencias}

Anuario N 29 (2014) visitado en http://www.unsaac.edu.pe/oficinas/estadistica/documentos

Bekker, I. (2004). An attitude scale for measuring language attitudes at South African tertiary institutions. Southern African Linguistics and Applied Language Studies Volume 22, Issue 1-2, 43-62.

Blanco, H. (1974). Tierra o muerte. Siglo Veintiuno Editores.

Bourdieu, P. (1989). O poder simbólico.

Voluntariado Hatun Nan [ca. 2017]. In Facebook [página de grupo]. Visto en April 16, 2017, de https://www.facebook.com/groups/249606725429517/

Cerrón-Palomino, R. (2003). Castellano andino: Aspectos sociolingüísticos, pedagógicos y gramaticales (1. ed.). Lima: Pontifícia Universidad Católica del Perú, Fondo Editorial.

Coronel-Molina, S. M. (2007). Language policy and planning, and language ideologies in Peru: The case of Cuzco's High Academy of the Quechua Language (Qheswa simi hamut'ana kuraq suntur). University of Pennsylvania.

CID-UNSAAC (Abril 15, 2017). Visitado en http://idiomas.unsaac.edu.pe/

Crystal, D. (1997). A dictionary of linguistics and phonetics, 4th ed. Oxford, UK: Blackwell. 
Hornberger, N. H. (1988). Bilingual education and language maintenance: A southern Perúvian Quechua case (Vol. 4). Walter de Gruyter.

Howard, R. (2007). Por los linderos de la lengua: Ideologías lingüísticas en los Andes (Vol. 26). Univ Catolica Perú.

Lauer, M. (1997). Andes imaginarios : Discursos del indigenismo 2 Cusco: Centro de Estudios Rurales Andinos Bartolomé de las Casas.

López, L. E. (2009). Interculturalidad, educación y ciudadanía : Perspectivas latinoamericanas (1. ed.). La Paz, Bolivia: FUNPROEIB Andes.

Manley, M. (2008). Quechua language attitudes and maintenance in Cuzco, Peru. Language Policy Quartely, 7 (4), 323-344.

Pacheco, K. (2007). Incas, indios y fiestas: reivindicaciones y representaciones en la configuración de la identidad cusqueña. Instituto Nacional de Cultura, Dirección Regional de Cultura de Cusco.

Pérez, B. (2006). Turismo y representación de la cultura: identidad cultural y resistencia en comunidades andinas del Cusco. Anthropologica, 24(24), 29-49.

Supa Huamán, H. (2010). Hilos de mi vida (Segunda edición.). Lima: Ediciones del Congreso del Perú.

Yataco, M. (2012). Políticas de estado y la exclusión de las lenguas indígenas en el Perú. Droit et cultures. Revue internationale interdisciplinaire, (63), 110-142.

Zavala, V. (2011). Racialization of the bilingual student in higher education: A case from the Perúvian Andes. Linguistics And Education, 22(4), 393-405. doi:10.1016/j.linged.2011.08.004 